\title{
CHANGES IN EMG-EMG COHERENCE DURING HAND GRASP MOVEMENTS
}

\author{
GAOXIANG OUYANG, ZHAOJIE JU and HONGHAI LIU* \\ Intelligent Systems \& Biomedical Robotics Group, School of Creative Technologies, \\ University of Portsmouth, England, PO1 2DJ, UK \\ "honghai.liu@port.ac.uk
}

Received 27 Augest 2013

Accepted 20 December 2013

\begin{abstract}
It is challenging to recognize human hand grasp movements through electromyogram (EMG) signals. In this paper, EMG-EMG coherence measure is used to describe correlations of EMG recordings during different hand grasp movements. Meanwhile, linear discriminant analysis (LDA) is utilized to evaluate the performance of the EMG-EMG coherence measure for identifying the grasp movements. Experimental results show that the EMG-EMG coherence measure is effective to extract correlations among EMG recordings, with which different types of hand grasp movements have been successfully distinguished. It is shown that the EMG-EMG coherence measure might be a potential tool to reveal the EMG-EMG correlation between the intermuscular interactions during hand grasp movements.
\end{abstract}

Keywords: EMG-EMG coherence; hand grasp; linear discriminant analysis.

\section{Introduction}

The electromyogram (EMG) signal is a measure of the summed activity of a number of motor unit action potentials (MUAP) lying in the vicinity of the recording electrode, ${ }^{1}$ which contains rich information about users' motion intention and thus can be utilized in the control of rehabilitation devices and assistive robots, such as the prosthetic hand or exoskeleton. ${ }^{2,3}$ Assiduous efforts have been made in recent years to improve classification accuracy of EMG signals during human hand movements. ${ }^{4,5}$ Current tendency of EMG-based prosthetic hand is to enable users to perform complex grasps or manipulations with natural muscle movements. ${ }^{6,7}$

To use EMG signals as command and/or control signals of prosthetic hand, a number of methods have been proposed to extract valuable information from EMG signals ranging from traditional linear methods such as time and frequency analysis to nonlinear methods. ${ }^{8}$ Graupe et al. used an autoregressive (AR) model to represent EMG signals, and motions were determined based on the parameters of the AR model. ${ }^{9}$ Bu et al. proposed a system using five EMG electrodes and a combination of Bayesian and neural networks to classify both location and motion in a cooking task. ${ }^{10}$ Chu et al. used a wavelet packet transform to extract a feature vector form EMG signals and a combination of multilayer perceptron network to classify nine hand motions. ${ }^{11}$ On the other hand, 
Tenore et al. proposed a system using traditional time-domain features of EMG signals and a multilayer perceptron as a classifier, which can identify ten finger movements with the recognition greater than $90 \%{ }^{12} \mathrm{Ju}$ et al. investigated and evaluated nonlinear approaches to extract EMG signal features and to identify different types of manipulation motions including different hand grasps and in-hand manipulations. ${ }^{13}$ The experimental results demonstrated the proposed nonlinear measures provide important supplemental information and the combination of linear and nonlinear measures of EMG signals could achieve the highest recognition rate of $96.7 \%$.

To some extent, results of these experiments indicate that hand movements can be identified by a system consisting of EMGs and a classifier. ${ }^{14}$ There are still some shortcomings in aforementioned studies, which have generally focused on the EMG activity of a single agonist muscle. However, hand grasping and manipulation often involve the recruitment of multiple muscles acting across multiple joints. The intermuscular interaction plays an important role in the hand grasp movements. ${ }^{15}$ More recently, coherence measures derived from pairs of EMGs recorded over synergist muscles (i.e., EMG-EMG coherence) have been used and are thought to provide a more representative measure of rhythmic activity across muscles. ${ }^{16,17}$ Farmer et al. found significant coherence in the frequency band from 16 to $32 \mathrm{~Hz}$ that appears related to specific motor parameters. ${ }^{18}$ Baker et al. found that coherence between cortical neurons and EMGs was modulated in a task-dependent manner from 20 to $30 \mathrm{~Hz}$ in primates performing precise hand tasks and that EMG-EMG coherence from intrinsic hand and forearm muscles was modulated similarly in humans performing the same task. ${ }^{19}$ Coherence can be used as an index of muscle coordination between pairs of muscles by identifying the strength and periodicity of common frequency characteristics between two EMG signals. ${ }^{20}$ Motivated by the merits of EMG-EMG coherence analysis, this study aims to explore whether the coherence measure can be effectively used to represent the intermuscular interactions during hand grasp movements.

\section{Materials and methods}

Eight ( 2 female, 6 male) healthy right-handed subjects were volunteered for this study. Their ages range from 23 to 40 and average is 32.5 years; body height average is 175.5 $\mathrm{cm}$; body mass average is $70 \mathrm{~kg}$. All participants gave informed consent prior to the experiments according to the University of Portsmouth CCI Faculty Ethics Committee.

\subsection{Experimental Procedure}

The experiment consists of both freely and different grasp gestures. Each type of grasps is repeated 10 times. Every motion lasts about 2 seconds. Between every two repetitions, participants had to relax the hand for 2 seconds in the intermediate state which is opening hand naturally without any muscle contraction. Once one motion with ten repetitions was finished, participants had to relax the hand for 2 minutes before the next motion started. This was designed to overcome the effects of muscle fatigue. 


\subsection{Data Collection}

The two EMG electrodes are applied to the subject's right forearm muscles, i.e. flexor pollicis longus (FPL) and flexor digitorum profundus (FDP). The sEMG data are recorded using DataLINK system from Biometrics LTD with a gel-skin contact area of about $4 \mathrm{~cm} 2$ for each bipolar electrode and a centre to centre recording distance of 20 $\mathrm{mm}$. The sampling frequency of DataLINK system in our experiment was set to be $1000 \mathrm{~Hz}$ and sEMG signals were amplified 1000 times and bandwidth is 10 to $300 \mathrm{~Hz}$ using a sEMG amplifier (SX230FW sEMG Amplifier, Biometrics LTD). To obtain goodquality signals, subjects were scrubbed with alcohol and shaved if necessary and then electrodes were applied over the body using the die cut medical grade double sided adhesive tape. Electrodes locations were selected according to the Musculoskelet of these four muscles and confirmed by muscle specific contractions, which include manually resisted finger flexion, extension and abduction. The captured sEMG signals were visualized on a computer screen giving participants feedback to choose the positions of electrodes with stronger sEMG signals.

To investigate the pattern of EMG-EMG coherence during different grasp gestures, EMG signals are selected and dissected from no action (dataset I), Grasp a disc using two fingers (dataset II), Grasp a can with three fingers (dataset III), and Grasp a ball with five fingers (dataset IV) intervals. In this study, 80 2-channel 1-sec EMG epochs are selected for each dataset. Short $(1-\mathrm{sec})$ EMG signals are used since the duration of the grasp gesture is only about a few seconds.

\subsection{Coherence analysis}

Coherence provides the cross-correlation between two sEMG signals at each frequency of the signals. It ranges from 0 to 1 with higher value representing higher correlation. In this study, Capon's nonparametric spectral estimation which is known as the "minimum variance distortionless response" (MVDR) with window length of 100 points and frequency resolution of $1 \mathrm{~Hz}$ was used. ${ }^{21}$ MVDR spectral estimation is based on the output of a bank of filters where the bandpass filters are both data and frequency dependent in comparison to parametric periodogram approach, which is both data and frequency independent. ${ }^{22}$ The details of MVDR can be found in Benesty et al. 2005. ${ }^{21}$

\subsection{Statistical analysis}

Significant differences in EMG-EMG coherece values at the specified frequency among the different hand grasp movements are tested with one-way ANOVA with Scheffe's post-hoc test. Our a priori hypothesis is that hand grasp would cause an increase in coherence levels. Differences were considered to be statistically significant at $\mathrm{P}<0.05$. 


\subsection{Linear Discriminant Analysis}

LDA is adopted to evaluate the capability and effectiveness of the coherence measures in classifying different hand grasp movements. The basic idea of LDA is to project highdimensional data onto a low-dimensional space such that data are reshaped to maximize the class separability. ${ }^{23}$ Consider a classification problem with $K$ classes $(K \geq 2)$. Suppose the data contain $M$ observations $\left(x_{i}, y_{i}\right), i=1,2, \cdots, M$, with $x_{i} \in R^{p}$ and $y_{i} \in\{1,2, \cdots, K\}$. LDA is to find the linear combination $c^{\prime} x$ which maximizes the ratio of $c^{\prime} B c / c^{\prime} W c$, which $B$ and $W$ denote the $p \times p$ between-class and within-class covariance matrices, respectively, defined by:

$$
B=\sum_{k=1}^{K} \frac{n_{k}}{N}\left(\mu^{(k)}-\mu\right)\left(\mu^{(k)}-\mu\right)^{T}
$$

and

$$
W=\sum_{k=1}^{K} \frac{n_{k}}{N} \hat{\Sigma}_{k}
$$

where $\mu^{(k)}$ and $\hat{\Sigma}_{k}, k=1,2, \cdots, K$, are the sample means and covariance matrices of each class (with $n_{k}$ samples) and $\mu$ is the total sample mean vector. The maximization problem in LDA is equivalent to solving the eigenproblem: $\left(W^{-1} B-\lambda I\right) c=0$. Thus, if $W$ is a non-singular matrix, Fisher's criterion is maximized when the projection matrix $c$ is composed of the eigenvectors of $W^{-1} B$ with at most $K-1$ corresponding non-zero eigenvalues. $^{23}$

\section{Results}

The MVDR method is used to estimate the EMG-EMG coherence between the FPL and FDP muscles during the diferrent hand grasp movements. In this study, the window length $L$ is selected as 100; the sampling frequency of EMG recordings is $1000 \mathrm{~Hz}$; and the resolution parameter $\mathrm{K}$ is set to be 500. Therefore, the EMG power spectrum is computed with the resolution of $1 \mathrm{~Hz}$. As an example, the raw EMG recordings and their corresponding power spectra are presented in Fig. 1. An evident increase in the EMG amplitude and in a wide frequency range of EMG power spectrum is observed in both FPL and FDP muscles, during the hand grasp an object. Then, based on the EMG power spectrum and EMG-EMG cross power spectrum between the FPL and FDP muscles, the EMG-EMG coherence can be obtained. As shown in Fig. 2, the values of EMG-EMG coherence from hand grasp an objective is much higher than those from no action in a wide frequency range. 

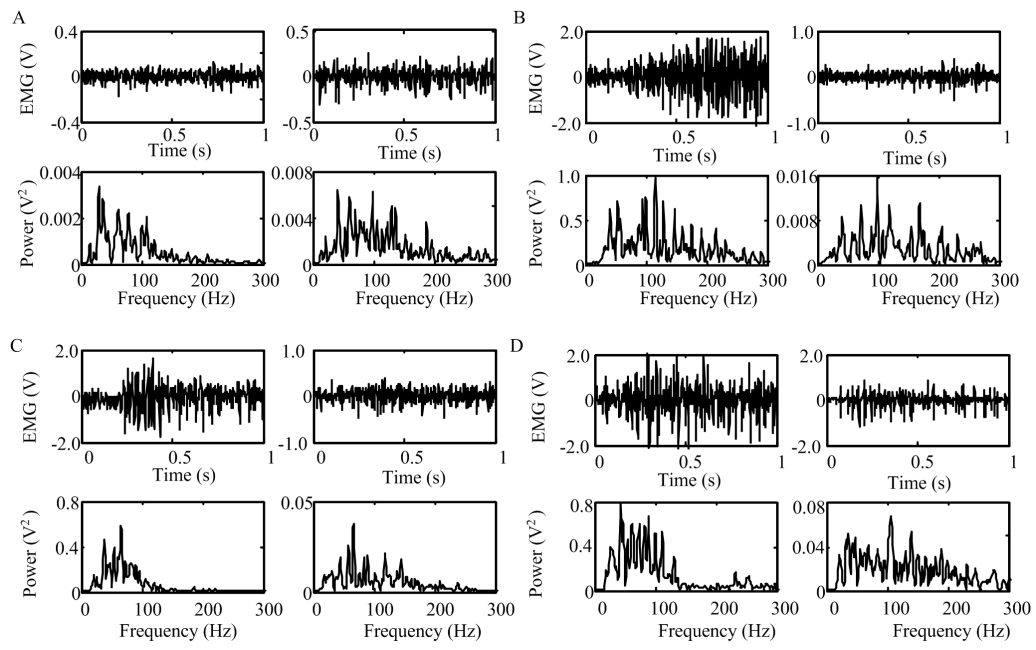

Fig. 1. Examples of raw EMG recordings from the muscles FPL (left) and FDP (right) and their corresponding power spectrum for four different hand movements, i.e. the no action (A), grasp a disc (B), grasp a can (C), and grasp a ball (D).
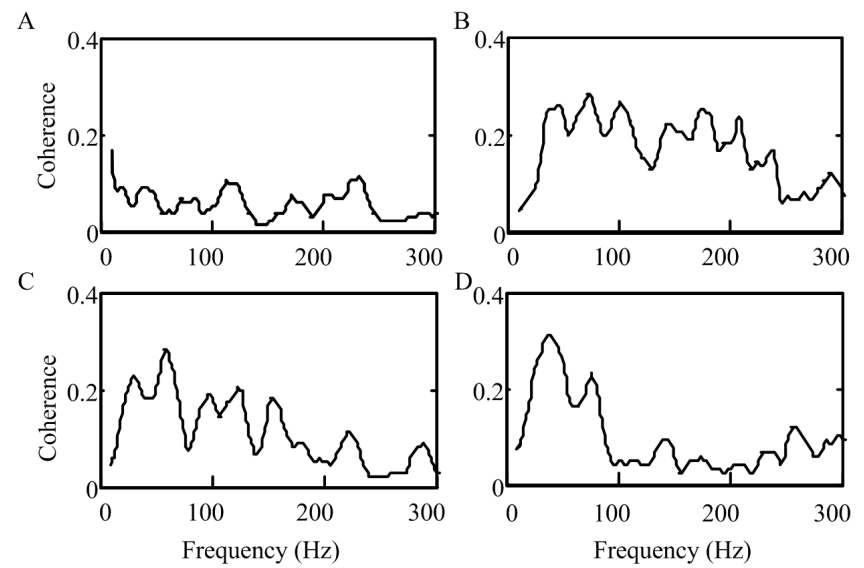

Fig. 2. Patterns of EMG-EMG coherence over the frequency range 10-300 $\mathrm{Hz}$ during four different hand movements, i.e. the no action (A), grasp a disc (B), grasp a can (C), and grasp a ball (D).

Next, the MVDR method is applied to analysing all EMG data. The mean EMGEMG coherence between the FPL and FDP muscles are presented in Fig. 3. It is found that the pattern of EMG-EMG coherence is changed for different hand grasp movements. A significant increase in EMG-EMG coherence during grasp a disc, when compared with the no action, is observed in the most frequency range 10-300 $\mathrm{Hz}$ (gray areas in Fig. 3A). The values of EMG-EMG coherence showed a significant increase in the frequency range $10-137,152-165$ and $168-184 \mathrm{~Hz}$ from no action to grasp a can (gray areas in Fig. 3B) 
and a significant increase in frequency range $10-138 \mathrm{~Hz}$ from no action to grasp a ball (gray areas in Fig. 3C) but there was no significant changes in the higher frequency band. Major headings should be typeset in boldface with the first letter of important words capitalized.
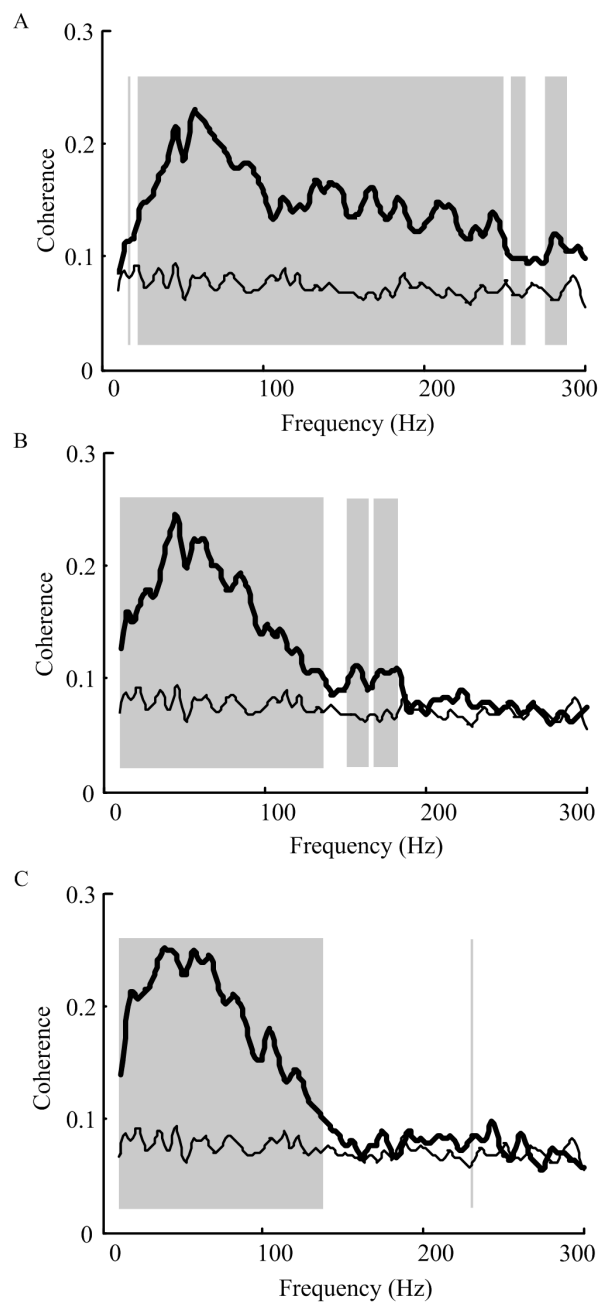

Fig. 3. Mean EMG-EMG coherence during the no action (line) and during the hand grasp movements (thick line), including grasp a disc (A), grasp a can (B), and grasp a ball (C). Grey areas represent the significant differences $(\mathrm{p}<0.05)$ between the EMG-EMG coherence at the corresponding frequency.

Then, in order to investigate whether their distributions over the four groups are significantly different, the one-way ANOVA with Scheffe's post-hoc test is used for mean coherence values on the frequency bands $10-30 \mathrm{~Hz}, 31-60 \mathrm{~Hz} 61-120 \mathrm{~Hz}, 121-200$ $\mathrm{Hz}$, and 200-300 Hz, respectively. The population distribution of the coherence of each frequency band is shown in Fig. 4 as boxplot. At the frequency band 10-30 Hz, it is found 
that there is significant difference among the EMG-EMG coherence values during four diferrent hand movements. Moreover, a gradual increase in EMG-EMG coherence is observed from the no action, grasp a disc, grasp a can, to grasp a ball. At the frequency band $31-60 \mathrm{~Hz}$ and $61-120 \mathrm{~Hz}$, it can also be found that the mean coherence values during the no action has significantly lower values than those during grasp a disc, grasp a can, and grasp a ball. However, the differences of coherence values can not be distinguished among the hand movements of grasp a disc, grasp a can and grasp a ball. Similar statistical results can also be obtained from the higher frequency bands, 120-200 $\mathrm{Hz}$ and 201-300 Hz, at which the differences of coherence values can not be distinguished among the hand grasp movements.
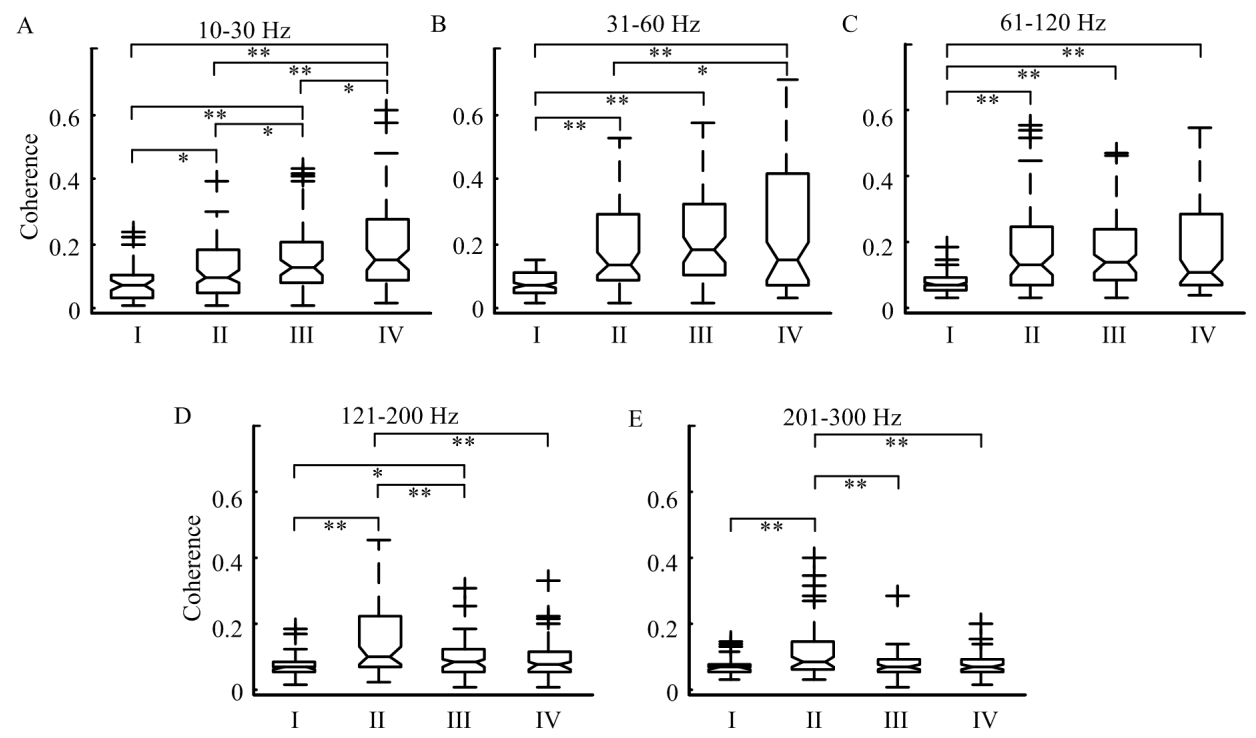

Fig.4. Boxplots for the EMG-EMG coherence at the specified frequency bands, grouped by the no action (I), grasp a disc (II), grasp a can (III), and grasp a ball (IV). Asterisks represent p-values resulting from a pair-wise comparison of the EMG-EMG coherence. Single asterisk $\left(^{*}\right)$ represents $\mathrm{p}<0.05$; double asterisk $\left({ }^{* *}\right)$ represents $\mathrm{p}<0.01$.

Finally, the ability and effectiveness of the above coherence measures in classifying the different hand grasp movements is evaluated using a LDA algorithm. The calculated coherence measures are used as input data with 291 features (dimension of the extracted feature vectors - coherence on frequency range 10 to $300 \mathrm{~Hz}$ ) in the LDA classifier. As shown in Fig. 5, these features are projected onto a two-dimensional space and the data are separated into well-defined clusters. The classification results are illustrated in Table 1, which show that LDA correctly classifies 313 out of 320 subjects, giving approximately $97.8 \%$ separability. 


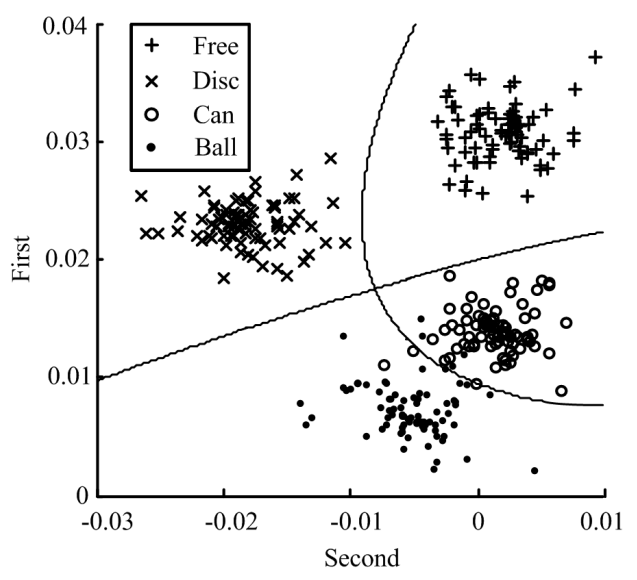

Fig. 5. Linear discriminant analysis of four groups, i.e, the no action, grasp a disc, grasp a can, and grasp a ball. The high dimensional feature vectors are projected down to two dimensions.

Table 1. Classification results with EMG-EMG coherence measures

\begin{tabular}{ccccc}
\hline Desired result & $\begin{array}{c}\text { Ouput result } \\
\text { Free }\end{array}$ & Disc & Can & Ball \\
\hline Free & 80 & 0 & 0 & 0 \\
\hline Disc & 0 & 80 & 0 & 0 \\
\hline Can & 0 & 0 & 77 & 3 \\
\hline Ball & 0 & 0 & 4 & 76 \\
\hline
\end{tabular}

\section{Conclusions}

In this study, we have analyzed the EMG-EMG correlations between EMG signals during different hand grasp movements using the MVDR coherence method. It is found that the values of EMG-EMG coherence from hand grasping an objective are significant higher than those from no action in a wide frequency range. Moreover, our results have shown that there is a significant difference in the pattern of EMG-EMG coherence between the FPL and FDP muscles during different hand grasp movements. Finally, the LDA classifier is applied to evaluate the performance of EMG-EMG coherence measures to discriminate among four hand grasp movements. A total classification accuracy of $97.8 \%$ is achieved. These results suggest that the EMG-EMG coherence measure has potential in identifying the EMG signals from different hand grasp movements.

\section{Acknowledgments}

This work was supported in part by U.K. National Engineering and Physical Scientific Research Council under Grant EP/G041377/1, State Key Lab of Digital Manufacturing Equipment \& Technology, China under Grant DMETKF2013001 and Leverhulme Trust under Grant 13754. 


\section{References}

[1] J. S. Karlsson, K. Roeleveld, C. Gronlund, A. Holtermann and N. Ostlund, Signal processing of the surface electromyogram to gain insight into neuromuscular physiology, Philos Transact A Math Phys Eng Sci 367 (2009) 337-56.

[2] H. Huang, Y. J. Pang, D. P. Yang, C. Y. Sun, L. Jiang, N. Li and H. Liu, A bio-mechanical designed prosthetic hand with multi-control strategies, International Journal of Humanoid Robotics 09 (2012) 1250013.

[3] D. Yang, J. Zhao, L. Jiang and H. Liu, Dynamic hand motion recognition based on transient and steadystate emg signals, International Journal of Humanoid Robotics 09 (2012) 1250007.

[4] H. S. Ryait, A. S. Arora and R. Agarwal Study of issues in the development of surface EMG controlled human hand, Journal of materials science. Materials in medicine 20 (2009) 107-114.

[5] H. H. Liu, Exploring Human Hand Capabilities into Embedded Multifingered Object Manipulation, IEEE Transaction on Industrial Informatics, 7 (2011) 389-398.

[6] Z. J. Ju, X. X. Zhu and H. H. Liu, Empirical copula-based templates to recognize surface EMG signals of hand motions, International Journal of Humanoid Robotics 8 (2011) 725-741.

[7] Q. Zhan and X. C. Liu, Hand grasp function analysis based on VF set, International Journal of Humanoid Robotics 10 (2013) 1350026

[8] C. Castellini and P. van der Smagt, Surface EMG in advanced hand prosthetics, Biol Cybern 100 (2009) 35-47.

[9] D. Graupe, J. Magnussen and A. A. Beex, Microprocessor system for multifunctional control of upperlimb prostheses via myoelectric signal identification, IEEE Transactions on Automatic Control 23 (1978) 538-544.

[10] N. Bu, M. Okamoto and T. Tsuji, A hybrid motion classification approach for EMG-based human-robot interfaces using bayesian and neural networks, IEEE Transactions on Robotics 25 (2009) 502-511.

[11] J. U. Chu, I. Moon and M. S. Mun, A real-time EMG pattern recognition system based on linear-nonlinear feature projection for a multifunction myoelectric hand, IEEE Trans Biomed Eng 53 (2006) 2232-2239.

[12] F. V. Tenore, A. Ramos, A. Fahmy, S. Acharya, R. Etienne-Cummings and N. V. Thakor, Decoding of individuated finger movements using surface electromyography, IEEE Trans Biomed Eng 56 (2009) 14271434.

[13] Z. J. Ju, G. X. Ouyang, M. Wilamowska-Korsak and H. H. Liu, Surface EMG based hand manipulation identification via nonlinear feature extraction and classification, IEEE Sensors Journal 13 (2013) 33023311.

[14] Z. J. Ju and H. H. Liu, Human Hand Motion Analysis With Multisensory Information, IEEE-ASME Transactions on Mechatronics, (2014) doi: 10.1109/TMECH.2013.2240312

[15] A. Schmied and M. Descarreaux, Reliability of EMG determinism to detect changes in motor unit synchrony and coherence during submaximal contraction, J Neurosci Methods 196 (2011) 238-246.

[16] 14. K. G. Keenan, J. D. Collins, W. V. Massey, T. J. Walters and H. D. Gruszka, Coherence between surface electromyograms is influenced by electrode placement in hand muscles, J Neurosci Methods $\mathbf{1 9 5}$ (2011) 10-14.

[17] K. G. Keenan, W. V. Massey, T. J. Walters and J. D. Collins, Sensitivity of EMG-EMG coherence to detect the common oscillatory drive to hand muscles in young and older adults, J Neurophysiol 107 (2012) 2866-2875.

[18] S. F. Farmer, J. Gibbs, D. M. Halliday, L. M. Harrison, L. M. James, M. J. Mayston and J. A. Stephens, Changes in EMG coherence between long and short thumb abductor muscles during human development, J Physiol 579 (2007) 389-402.

[19] S. N. Baker, J. M. Kilner, E. M. Pinches and R. N. Lemon, The role of synchrony and oscillations in the motor output, Exp Brain Res 128 (1999) 109-117.

[20] A. Danna-Dos Santos A, B. Poston, M. Jesunathadas, L. Bobich, T. Hamm and M. Santello, Influence of fatigue on hand muscle coordination and EMG-EMG coherence during three-digit grasping, $J$ Neurophysiol 104 (2010) 3576-3587.

[21] Benesty J., Chen J. D. and Huang Y. T. 2005, "A generalized MVDR spectrum," IEEE Signal Processing Letters 12, 827-830.

[22] D. Cvetkovic and I. Cosic, EEG inter/intra-hemispheric coherence and asymmetric responses to visual stimulations, Med Biol Eng Comput 47 (2009) 1023-1034.

[23] A. R. Webb, Statistical pattern recognition, 2nd edn. (Wiley, Chichester, United Kingdom, 2006) 